\title{
Hierarchical Self-Assembly of Supramolecular Double-Comb Triblock Terpolymers
}

\author{
Anton H. Hofman, ${ }^{\dagger, \dagger}$ Ivan Terzic, ${ }^{\dagger}$ Marc C. A. Stuart, ${ }^{\S, \|_{\odot}}$ Gerrit ten Brinke, ${ }^{\dagger}$ and Katja Loos ${ }^{*}{ }^{\dagger}(0)$ \\ ${ }^{\dagger}$ Macromolecular Chemistry and New Polymeric Materials, Zernike Institute for Advanced Materials, University of Groningen, \\ Nijenborgh 4, 9747 AG Groningen, The Netherlands \\ ${ }^{\S}$ Stratingh Institute for Chemistry, University of Groningen, Nijenborgh 4, 9747 AG Groningen, The Netherlands \\ "Electron Microscopy Group, Groningen Biomolecular Sciences and Biotechnology Institute, University of Groningen, Nijenborgh \\ 7, 9747 AG Groningen, The Netherlands
}

\section{Supporting Information}

ABSTRACT: Involving supramolecular chemistry in self-assembling block copolymer systems enables design of macromolecular architectures that are challenging to obtain through conventional all-covalent routes. In this work we present supramolecular double-comb triblock terpolymers in which both outer blocks are able to interact with a surfactant via hydrogen bonding and thereby form a comb-shaped architecture upon complexation. While the neat triblock terpolymer only formed a triple lamellar morphology, multiple hierarchical structures were observed in these supramolecular comb-coil-comb triblock terpolymers by simply adjusting the surfactant concentration. Structures included spheres on tetragonally packed cylinders-inlamellae and spheres on double parallel lamellae-in-lamellae, as evidenced by electron microscopy and X-ray scattering. Incorporation
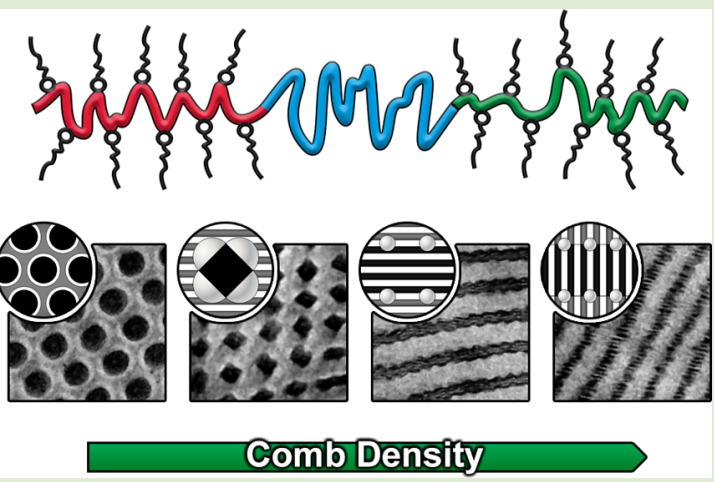
of a middle coil block thus allowed an even higher macromolecular complexity than the previously reported double-comb diblock copolymers.

B lock copolymers are able to assemble into a wide variety of nanometer-sized structures, with the type of structure depending on the molecular weight, composition, and architecture. ${ }^{1}$ Copolymers with a more complex macromolecular architecture often give rise to more complex selfassembly. ${ }^{2,3}$ While microphase separation of diblock copolymers is limited to spherical, cylindrical, lamellar and gyroid structures, ${ }^{4}$ over 30 different phases have been identified for linear triblock terpolymers. ${ }^{5}$ Examples include helical structures, ${ }^{6}$ core-shell gyroid morphologies, ${ }^{7}$ and tricontinuous double-diamond structures. ${ }^{8}$ For obtaining three-phase morphologies, but avoiding the challenging synthesis that is accompanied by the preparation of terpolymers, several research groups have investigated the phase behavior of copolymer blends, either with interacting ${ }^{9-11}$ or noninteracting polymer pairs. $^{12-15}$

An alternative approach is the use of surfactants that associate with a certain polymer through hydrogen bonding or electrostatic interactions, thereby resulting in a supramolecular comb-shaped copolymer. ${ }^{16}$ One of the most studied H-bonded comb copolymers is based on poly(4-vinylpyridine) (P4VP) and 3-pentadecylphenol surfactants that forms a layered crystalline structure at room temperature, transforms into liquid crystalline lamellae on heating, and finally gives a disordered melt at sufficiently high temperatures. ${ }^{17}$ The exact transition temperatures depend on the tail length and substitution of the alkylphenol. ${ }^{18}$ When this supramolecular system was combined with polystyrene-containing (PS) diblock copolymers, simultaneous self-assembly of the comb block and PS- $b$-P4VP diblock led to the formation of hierarchical morphologies, that is, structures within another structure. ${ }^{19}$ Adjustment of the block copolymer composition ${ }^{20}$ or the surfactant concentration ${ }^{21}$ gave access to a wide variety of structures that had not been observed in all-covalent systems. Surfactants have been combined with triblock terpolymers that contained a single hydrogen bond accepting block, although in these examples, the structure within the comb block remained unresolved. ${ }^{22,23}$

This supramolecular approach was recently extended to double-comb diblock copolymer complexes by exchanging the PS block for an acrylamide block that could form a supramolecular complex as well, thus resulting in hierarchical morphologies in both polymer phases. $^{24,25}$ In symmetric diblock copolymer systems, highly unusual double parallel ${ }^{26}$ and double perpendicular ${ }^{27}$ lamellae-in-lamellae were observed over a wide range of molecular weights. In the asymmetric complexes, however, crystallization of the surfactant and the

Received: July 28, 2018

Accepted: September 6, 2018

Published: September 13, 2018 
Scheme 1. (a) Chemical Structure and (b) Schematic Representation of the [P4VP-b-PS- $b$-PAPI] (3-NDP) Supramolecular Double-Comb Triblock Terpolymer

a)

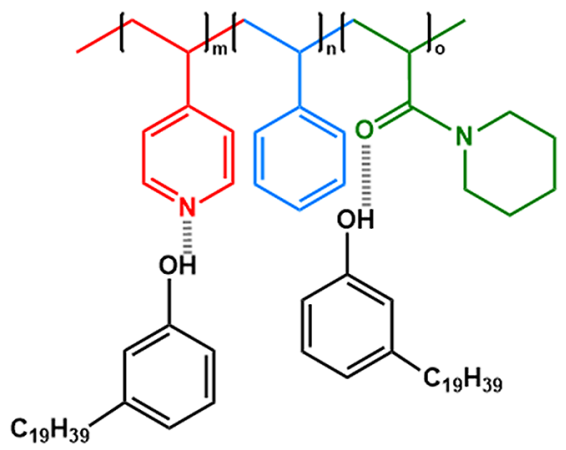

b)

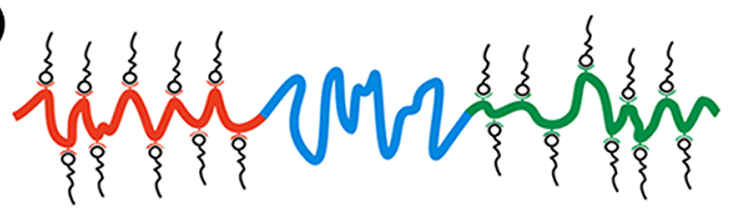

tendency of the double-comb to avoid the formation of a curved interface disturbed formation of ordered structures. ${ }^{28}$

In this work we demonstrate self-assembly of the first double-comb triblock terpolymer by inserting a PS coil block in between the P4VP and poly( $N$-acryloylpiperidine) (PAPI) blocks. Addition of 3-nonadecylphenol (3-NDP) resulted in the formation of a [P4VP- $b$-PS- $b$-PAPI](3-NDP) $)_{x}$ supramolecular comb-coil-comb triblock terpolymer (Scheme 1), where $x$ denotes the number of 3-NDP molecules per monomer unit, but excluding the PS block; this notation enables direct comparison with the diblock system. By employing a single P4VP-b-PS- $b$-PAPI triblock terpolymer and adjusting the concentration of surfactant, we found multiple hierarchical morphologies that have not been observed in block copolymer-based materials before.

The P4VP- $b$-PS- $b$-PAPI triblock terpolymer used for this study was synthesized through RAFT polymerization $\left(M_{\mathrm{n}}=\right.$ $\left.84.1 \mathrm{~kg} \mathrm{~mol}^{-1}, Ð=1.08\right)^{29}$ and had similar weight fractions for all three blocks $\left(f_{\mathrm{P} 4 \mathrm{VP}}=0.34, f_{\mathrm{PS}}=0.28, f_{\mathrm{PAPI}}=0.38\right.$; Figure S1). Transmission electron microscopy (TEM) demonstrated this terpolymer (P4PSPA84k) to self-assemble into a triple lamellar morphology (Figure 1a), with this TEM micrograph being representative for the bulk material as evidenced by small-angle X-ray scattering (SAXS) (Figure $1 \mathrm{~b}$ ). Up to five scattering maxima with an integer ratio between the first and
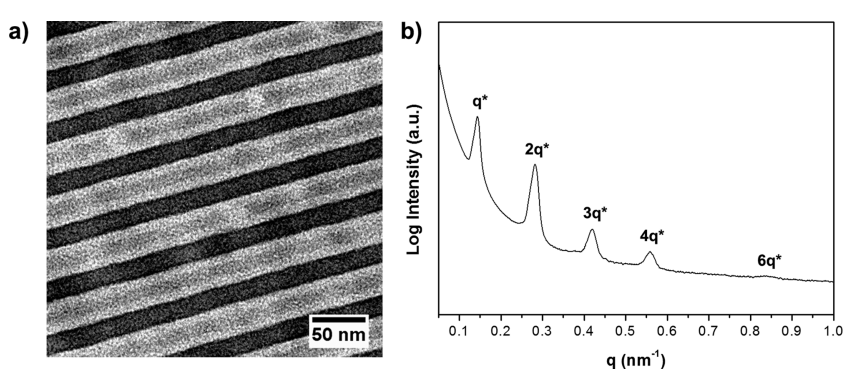

Figure 1. (a) TEM image of the neat P4VP-b-PS-b-PAPI triblock terpolymer. P4VP appears dark due to staining with iodine, PAPI is gray, and PS is white. (b) SAXS pattern recorded at room temperature. higher order reflections can be observed, implying a lamellar periodicity of $43.5 \mathrm{~nm}$. The structure appeared to be highly ordered, as single grains covered several microns (Figure S2). Triple lamellae would indeed be the expected structure, as such an equilibrium morphology was found for both frustrated ${ }^{30}$ and nonfrustrated ${ }^{31}$ terpolymers in which the three blocks are of equal length.

Four supramolecular complexes were prepared by adjusting the concentration of surfactant $(x=0.1-1.0)$ and were subsequently analyzed by differential scanning calorimetry (DSC). Since the thermal properties of the terpolymer complexes were found to be unaffected by inclusion of a PS middle block (Figure S3), ${ }^{25}$ it can be concluded that the distribution of 3-NDP over the P4VP and PAPI H-bonding phases does not differ from the diblock copolymer system: for low values of $x$ (0.1 and 0.3) 3-NDP favors PAPI, whereas at higher concentrations (0.5 and 1.0) it is approximately evenly distributed.

The glass transition of $\mathrm{P} 4 \mathrm{VP}$ reduced from $151^{\circ} \mathrm{C}$ to $139^{\circ} \mathrm{C}$ in P4PSPA84k(3-NDP) $)_{0.1}$, implying the majority of $3-\mathrm{NDP}$ being $\mathrm{H}$-bonded to PAPI; symmetric distribution would have resulted in a $T_{\mathrm{g}}$ as low as $91{ }^{\circ} \mathrm{C}$. Thin sections analyzed by TEM demonstrated this complex to self-assemble into a highly ordered core-shell cylindrical morphology (Figure 2a,b). This phase transition confirms the highly asymmetric surfactant distribution: the cylinders are formed by 3-NDP-poor P4VP, the shells by PS and the matrix by 3-NDP-rich PAPI. Although fast Fourier transforms (FFTs) of these images suggest hexagonal packing (Figure S4), additional information on the complex's symmetry was obtained through temperatureresolved SAXS (Figures S5a and S6a). ${ }^{32,33}$ At room temperature the occurrence of scattering maxima with a ratio of $q^{*}: \sqrt{3} q^{*}: \sqrt{7} q^{*}: \sqrt{9} q^{*}: \sqrt{19} q^{*}$ confirm hexagonal packing ( $d=$ $49.4 \mathrm{~nm}$ ), while the complex remained unaffected by the temperature (Figure 3). Scattering at higher $q$-values was absent and without applying a staining agent no features can be detected within the matrix (Figure 4a), indicating the concentration of surfactant being insufficiently high to form an internal structure.

The core-shell structure found for this supramolecular complex is a typical example of a type 1 frustrated triblock copolymer system. ${ }^{34-36}$ Detailed analysis of the frustration is impossible, as inclusion of surfactant alters the effective polymer incompatibility. ${ }^{37}$ However, since P4VP only contains a minimal amount of 3-NDP, the most unfavorable P4VP/PS interface $^{38}$ remains practically unaffected and is minimized through such a transition (Scheme 2a).

Similar to the $x=0.1$ supramolecular complex, a highly asymmetric surfactant distribution caused P4PSPA84k(3$\mathrm{NDP})_{0.3}$ to self-assemble into a cylindrical morphology (Figure 2c). The 3-NDP-poor P4VP cylindrical microdomains seem rectangular and a bright spherical PS substructure $z$ can be observed, that can be more clearly identified in other regions of the sample (Figures 2d and S7a). The FFT of this image (Figure S7b) confirms the PS spheres to be evenly distributed along the cylinder axis with an interspherical distance $d_{z} \approx 16$ $\mathrm{nm}$. Uneven distribution of the surfactant, with its preference for PAPI, allowed 3-NDP to crystallize inside the matrix $\left(T_{\mathrm{m}}=\right.$ $30{ }^{\circ} \mathrm{C}$, Figure S3) and form small layers in both a parallel and perpendicular orientation (Figure 4b). Room-temperature SAXS measurements confirmed the presence of both the spherical structure $\left(d_{z}=11.8 \mathrm{~nm}\right)^{10}$ and the smaller surfactantbased structure $\left(d_{\mathrm{s}}=4.1 \mathrm{~nm}\right)$, whereas absence of multiple 
a)

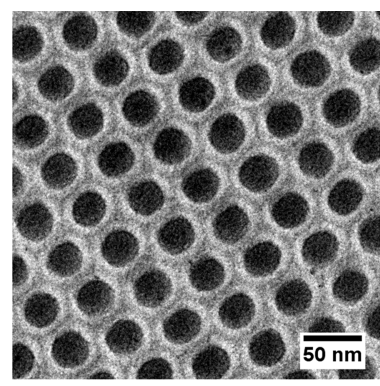

b)

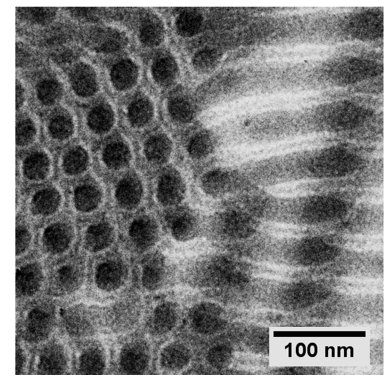

c)

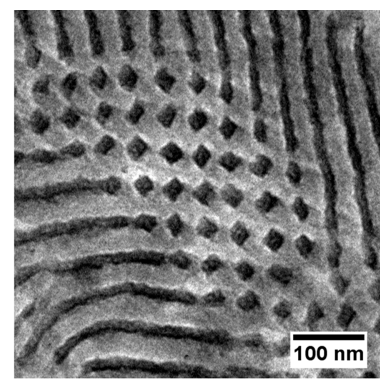

e)

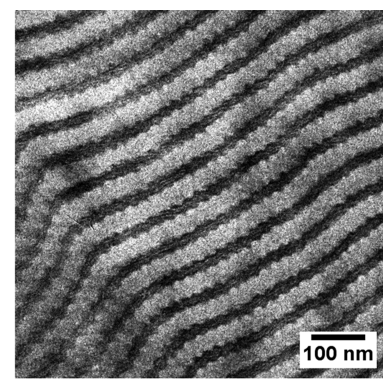

g)

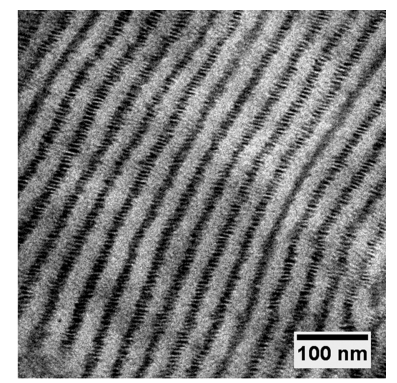

d)

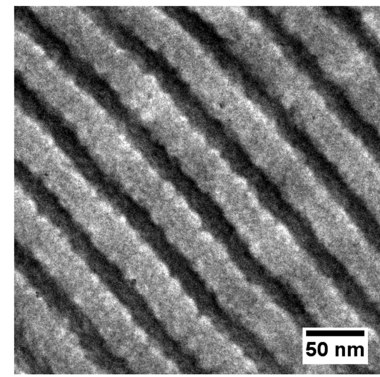

f)

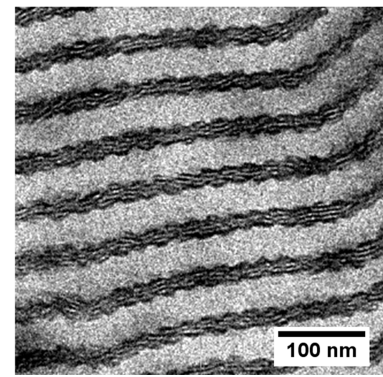

h)

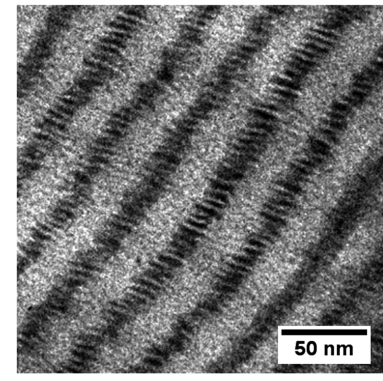

Figure 2. TEM images of iodine-stained sections of the P4PSPA84k(3-NDP) $)_{x}$ supramolecular double-comb triblock terpolymer complexes, with $x$ $=0.1(\mathrm{a}, \mathrm{b}), x=0.3(\mathrm{c}, \mathrm{d}), x=0.5(\mathrm{e}, \mathrm{f})$, and $x=1.0(\mathrm{~g}, \mathrm{~h})$. P4VP appears dark, PAPI is gray, and PS is white.

higher order scattering maxima prevented identification of the large length scale morphology (Figure 3a).

Temperature-resolved SAXS enabled a detailed analysis of the equilibrium structure (Figure S5b). Although characteristic scattering of the small lamellae $\left(q_{\mathrm{s}}\right)$ diminished above the melting point of PAPI(3-NDP) (Figure 3b), all other scattering events remained in place, implying an unchanged block copolymer morphology $(d=57.0 \mathrm{~nm})$. The deviating shape of $q_{z}$ confirms this signal to originate from the PS substructure, whereas appearance of a peak at $\sqrt{8} q^{*}$ indicates tetragonal packing of the diamond-shaped cylinders. ${ }^{39}$ Fourier transformation of a perpendicularly ordered area in TEM indeed more closely resembles tetragonal than hexagonal packing (Figure S7c,d). Additional maxima appeared at low $q$
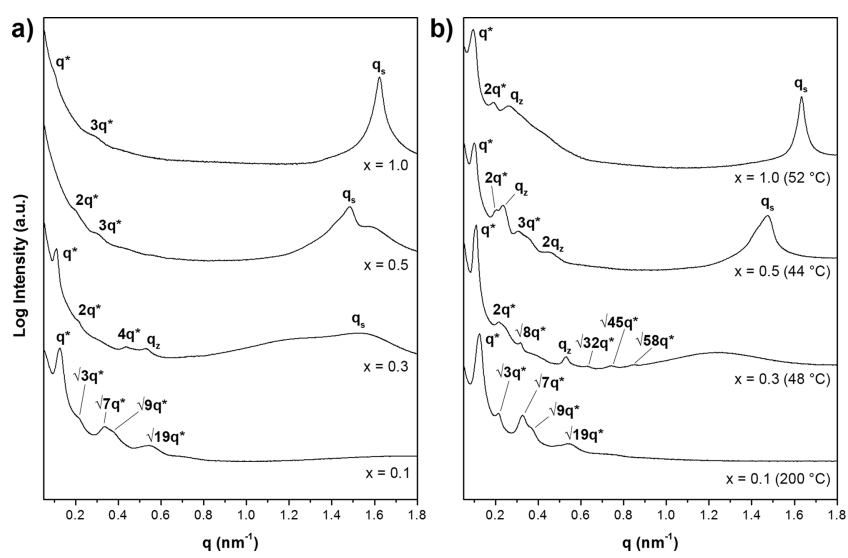

Figure 3. (a) SAXS profiles of the P4PSPA84k(3-NDP) $x$ supramolecular complexes recorded at room temperature and $(\mathrm{b})$ at the temperature indicated. First-order reflections $\left(q^{*}\right)$ were used to calculate the domain spacing $d$ of the large structure, $q_{z}$ indicates scattering of a PS substructure, and $q_{s}$ corresponds to a surfactantbased structure. on further heating supporting the tetragonal lattice (Figure S6b).

Rectangular and tetragonally packed cylindrical structures have been described for several block copolymer-based systems, ${ }^{40}$ including H-bonding diblock copolymer blends, ${ }^{10,41}$ triblock terpolymer blends, ${ }^{39,42,43}$ ionic supramolecular complexes, ${ }^{44,45}$ and wedge-shaped surfactant-containing systems. ${ }^{46}$ Increased conformational entropy, a reduced surface area or surfactant-directed packing are among the arguments that have been reported. None of these apply for our terpolymer system, as it is very monodisperse, there is no composition effect, linear surfactants were used and the orientation of the small lamellae is random. A plausible explanation for the formation of PS spheres on diamond-shaped cylinders-in-lamellae (Scheme 2b) is reduction of the unfavorable $\mathrm{P} 4 \mathrm{VP} / \mathrm{PS}$ interface in the rectangular situation compared to spheres on round P4VP cylinders, whereas a tetragonal lattice may allow a more advantageous packing of 3-NDP.

The P4PSPA84k(3-NDP) $)_{0.5}$ supramolecular complex displays two melting points $\left(T_{m, 1}=36^{\circ} \mathrm{C}\right.$ and $\left.T_{\mathrm{m}, 2}=44^{\circ} \mathrm{C}\right)$ and an additional phase transition at $77^{\circ} \mathrm{C}$ that corresponds to the order-disorder transition (ODT) of the P4VP(3-NDP) comb block (Figure S3). Although addition of 3-NDP to the triblock copolymer caused the large structure of this complex to remain lamellar, indicating approximately equal distribution of the surfactant, bright PS spheres are located in between the large lamellae, and parallelly oriented small lamellae can be observed inside P4VP (Figure 2e,f). FFT-analysis enabled estimation of the interspherical distance (Figure S8a,b, $d_{z} \approx 23 \mathrm{~nm}$ ). Despite the poor contrast, small lamellae can also be recognized inside PAPI without applying a staining agent (Figure 4c). Partial integration of this micrograph (Figure S8c) clearly showed a 4-5 $\mathrm{nm}$ periodic structure that covers both polymer phases (Figure S8d,e). The reduced order of the small lamellae compared to the diblock copolymer complexes $^{26}$ is likely caused by the glassy spherical PS microdomains.

Due to weak scattering of the complex at room temperature (Figure 3a), SAXS only allowed investigation of the small 
a)

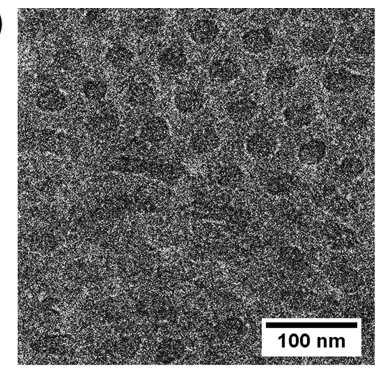

c)

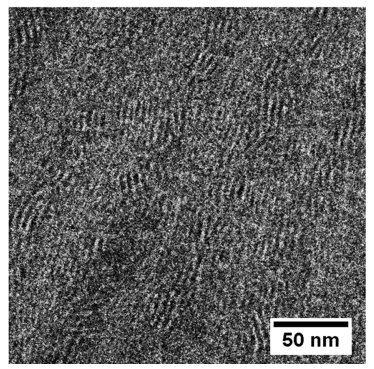

b)

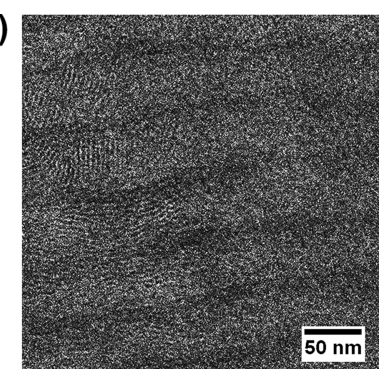

d)

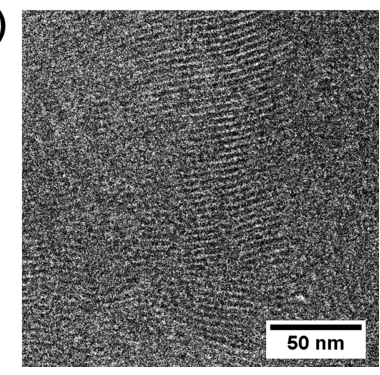

Figure 4. TEM micrographs of unstained P4PSPA84k(3-NDP) complexes with $x=0.1(\mathrm{a}), x=0.3(\mathrm{~b}), x=0.5(\mathrm{c})$, and $x=1.0$ (d). A smaller length scale morphology can be observed for $x=0.3-$ 1.0.

structure $\left(d_{\mathrm{s}}=4.2 \mathrm{~nm}\right)$. Two shoulders are visible in the low- $q$ region that will later appear to be a second and third order reflection $(d=62.8 \mathrm{~nm})$. Absence of the first-order maximum has been reported for triblock terpolymers in which the middle block has the lowest electron density and the outer blocks have a similar density. ${ }^{22,47}$ This argument also applies for P4PSPA84k(3-NDP) $)_{0.5}$, since both outer blocks almost exclusively contain 3-NDP.

As a function of temperature, the complex undergoes multiple phase transitions (Figure S5c) and indeed, $q^{*}$ became visible on melting of PAPI(3-NDP), while the positions of $2 q^{*}$ and $3 q^{*}$ remained unaffected (Figure $3 b$ ). Another strong scattering maximum appeared at $0.237 \mathrm{~nm}^{-1}$ that corresponds to the distance between the PS spheres $\left(d_{z}=26.7 \mathrm{~nm}\right)$ being in excellent agreement with the TEM results. Heating above the ODT of P4VP(3-NDP) resulted in disappearance of the small structure and a change of the packing symmetry of the PS spheres (Figure S6c). The large lamellae remained intact throughout the temperature scan, while the reducing intensity of $q_{z}$ and its final disappearance at $200^{\circ} \mathrm{C}$ provided additional evidence for these signals to originate from the spherical PS substructure. Alkylphenol-based surfactants indeed become miscible with PS at elevated temperatures, and therefore, the PS middle block will merge with the outer blocks, thereby giving rise to a simple large lamellar structure. ${ }^{48}$ Despite this complicated thermal behavior, at room temperature the triblock system closely resembles the double parallel lamellarin-lamellar morphology previously found in the diblock analogue, $^{26}$ but contains an additional PS spherical substructure (Scheme 2c).

The stoichiometric complex P4PSPA84k(3-NDP) $)_{1.0}$ selfassembled into highly ordered double perpendicular lamellaein-lamellae: with staining small layers can be recognized inside P4VP (Figures 2g,h and S9a), while large areas covered with small lamellae can be seen in the absence of staining agent (Figures $4 \mathrm{~d}$ and $\mathrm{S} 9 \mathrm{c}$ ), indirectly demonstrating the selfassembly of PAPI(3-NDP). PS domains are not visible under

both conditions due to the low concentration of PS $(<10 \mathrm{wt}$ $\%)$. Besides the small structure $\left(d_{\mathrm{s}}=3.9 \mathrm{~nm}\right)$, SAXS displays two shoulders at low $q$ (Figure 3a), enabling direct analysis of the large structure $(d=63.6 \mathrm{~nm})$. The polymer chains are thus highly stretched compared to the neat terpolymer.

The transition temperatures of P4PSPA84k(3-NDP) $)_{1.0}$ are identical to the stoichiometric homopolymer complexes, ${ }^{25}$ implying 3-NDP being equally distributed. Upon passing the melting point of PAPI $(3-\mathrm{NDP})\left(52^{\circ} \mathrm{C} ; \mathrm{T}_{\mathrm{m}, 1}=45^{\circ} \mathrm{C}\right)$, a broad scattering peak appeared at $0.262 \mathrm{~nm}^{-1}$, whereas both the small structure in P4VP and the large structure remained intact (Figure 3b). Its shape and position indicate this reflection to originate from a PS substructure, being almost identical to the $x=0.5$ supramolecular complex $\left(d_{z}=23.8 \mathrm{~nm}\right)$. The primary scattering peak disappeared in the liquid crystalline state $\left(68^{\circ} \mathrm{C} ; T_{\mathrm{m}, 2}=54^{\circ} \mathrm{C}\right.$ and $\left.T_{\mathrm{ODT}}=71^{\circ} \mathrm{C}\right)$ for similar reasons as discussed for P4PSPA84k(3-NDP) 0.5 (Figures S5d and S6d). On further heating, $q_{z}$ merged with $2 q^{*}$ and finally disappeared at $110^{\circ} \mathrm{C}$, because of PS becoming miscible with the other components. The temperature-dependent distribution of 3NDP caused the first-order scattering maximum to reappear at $137^{\circ} \mathrm{C}$. In this state, the supramolecular complex behaves as a solvent-swollen diblock copolymer and forms a simple lamellar morphology $(d=50.9 \mathrm{~nm})$. All reflections broadened on further heating and gradually disappeared around $190^{\circ} \mathrm{C}$, implying the formation of a disordered system. The microphase separated structure is thermally much more stable than the corresponding diblock copolymer system that already became disordered above the ODT of P4VP(3-NDP). ${ }^{27}$ On cooling from a disordered melt the PS block thus directs the self-assembly of P4PSPA84k(3-NDP) $)_{1.0}$, giving rise to enhanced contrast in SAXS and the formation of a highly

Scheme 2. Schematic Illustration of the Structures Identified in the Triblock Terpolymer Complexes: (a) Hexagonally Packed Core-Shell Cylinders $(x=0.1),(b)$ Spheres on Tetragonally Packed Diamond-Shaped Cylinders-in-Lamellae $(x=0.3)$, (c) Spheres on Double Parallel Lamellae-in-Lamellae $(x=0.5)$, and (d) Spheres on Double Perpendicular Lamellae-in-Lamellae $(x=1.0)^{a}$ a)

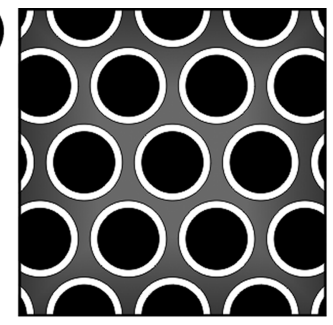

c)

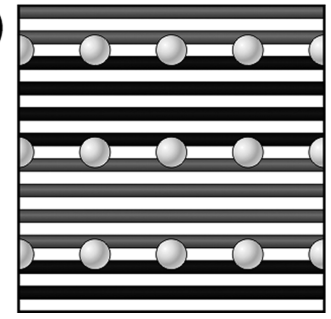

b)

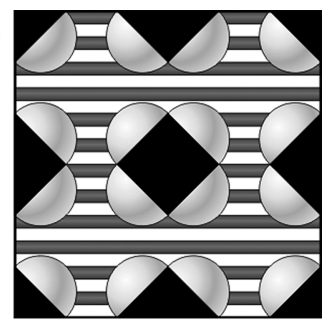

d)

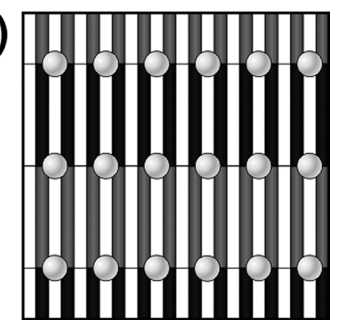

${ }^{a}$ Black corresponds to P4VP, dark grey to PAPI, white lamellae to 3NDP $(b-d)$, and white shells (a) or light grey spherical structures $(b-d)$ to PS. 
ordered layered structure. Strong scattering of the $z$-structure in the melt implies the presence of isolated PS (spherical) microdomains in between the perpendicular lamellae-inlamellae (Scheme 2d), although PS being dispersed in either PAPI(3-NDP) or P4VP(3-NDP) at room temperature should not be excluded, but seems unlikely. ${ }^{20,48}$

In this work we demonstrated the first example of hierarchically self-assembling supramolecular double-comb triblock terpolymers. Incorporation of a PS middle block resulted in significantly more complex phase behavior compared to the previously studied double-comb diblock copolymers. Already four new hierarchical morphologies were identified by using a single terpolymer and adjusting the comb density $x$ (Scheme 2). For $x=0.1,3-N D P$ only plasticized PAPI, and the changed volume fractions caused the complex to transform into a hexagonal core-shell cylindrical morphology. Spheres on tetragonally packed diamond-shaped cylinders were formed by increasing the amount of 3-NDP $(x=0.3)$, and allowed the surfactant to form small lamellae inside the 3NDP-rich matrix. Preference of 3-NDP for PAPI was no longer observed at higher grafting densities, resulting in PS spheres distributed along either double parallel $(x=0.5)$ or double perpendicular $(x=1.0)$ lamellae-in-lamellae. Even more complex phase behavior is expected to be achieved by varying either the composition or the block sequence. Additionally, inclusion of a larger PS middle block could result in freestanding networks that would enable design of hierarchically porous materials through selective surfactant removal, ${ }^{49}$ while the observed tetragonally packed supramolecular structures could be interesting templates in nanopatterning applications. ${ }^{41,43}$

\section{ASSOCIATED CONTENT}

\section{S Supporting Information}

The Supporting Information is available free of charge on the ACS Publications website at DOI: 10.1021/acsmacrolett.8b00570.

Experimental procedures, details on the synthesis and characterization of the triblock terpolymer, thermal analysis of the complexes, temperature-resolved SAXS measurements, TEM images recorded at a lower magnification and detailed analysis of the TEM data (PDF).

\section{AUTHOR INFORMATION}

\section{Corresponding Author}

*E-mail: k.u.loos@rug.nl.

ORCID

Marc C. A. Stuart: 0000-0003-0667-6338

Katja Loos: 0000-0002-4613-1159

\section{Present Address}

${ }^{\ddagger}$ Physical Chemistry and Soft Matter, Wageningen University, Stippeneng 4, 6708 WE Wageningen, The Netherlands.

\section{Notes}

The authors declare no competing financial interest.

\section{ACKNOWLEDGMENTS}

This work was funded by The Netherlands Organization for Scientific Research (NWO) via a VICI innovational research grant. Beam time on the Dutch-Belgian Beamline (DUBBLE) of ESRF (Grenoble, France) has kindly been made available by
NWO, and we would like to thank Daniel Hermida-Merino for his experimental assistance.

\section{REFERENCES}

(1) Abetz, V.; Simon, P. F. W. Phase behaviour and morphologies of block copolymers. Adv. Polym. Sci. 2005, 189, 125-212.

(2) Polymeropoulos, G.; Zapsas, G.; Ntetsikas, K.; Bilalis, P.; Gnanou, Y.; Hadjichristidis, N. 50th Anniversary Perspective: Polymers with Complex Architectures. Macromolecules 2017, 50, $1253-1290$.

(3) Bates, C. M.; Bates, F. S. 50th Anniversary Perspective: Block Polymers-Pure Potential. Macromolecules 2017, 50, 3-22.

(4) Faber, M.; Hofman, A. H.; Loos, K.; ten Brinke, G. Highly Ordered Structure Formation in RAFT-Synthesized PtBOS-b-P4VP Diblock Copolymers. Macromol. Rapid Commun. 2016, 37, 911-919.

(5) Hadjichristidis, N.; Iatrou, H.; Pitsikalis, M.; Pispas, S.; Avgeropoulos, A. Linear and non-linear triblock terpolymers. Synthesis, self-assembly in selective solvents and in bulk. Prog. Polym. Sci. 2005, 30, 725-782.

(6) Breiner, U.; Krappe, U.; Abetz, V.; Stadler, R. Cylindrical morphologies in asymmetric ABC triblock copolymers. Macromol. Chem. Phys. 1997, 198, 1051-1083.

(7) Löbling, T. I.; Hiekkataipale, P.; Hanisch, A.; Bennet, F.; Schmalz, H.; Ikkala, O.; Gröschel, A. H.; Müller, A. H. E. Bulk morphologies of polystyrene-block-polybutadiene-block-poly(tert-butyl methacrylate) triblock terpolymers. Polymer 2015, 72, 479-489.

(8) Wang, Y.; Inoue, A.; Hasegawa, H.; Takenaka, M. The Formation of OTDD Network Structure in PS- $b$-PI- $b$-PDMS Triblock Terpolymer. Macromol. Chem. Phys. 2017, 218, 1700008.

(9) Jiang, S.; Göpfert, A.; Abetz, V. Novel Morphologies of Block Copolymer Blends via Hydrogen Bonding. Macromolecules 2003, 36, 6171-6177.

(10) Asari, T.; Matsuo, S.; Takano, A.; Matsushita, Y. Three-Phase Hierarchical Structures from $\mathrm{AB} / \mathrm{CD}$ Diblock Copolymer Blends with Complemental Hydrogen Bonding Interaction. Macromolecules 2005, $38,8811-8815$.

(11) Miyase, H.; Asai, Y.; Takano, A.; Matsushita, Y. Kaleidoscopic Tiling Patterns with Large Unit Cells from ABC Star-Shaped Terpolymer/Diblock Copolymer Blends with Hydrogen Bonding Interaction. Macromolecules 2017, 50, 979-986.

(12) Abetz, V.; Goldacker, T. Formation of superlattices via blending of block copolymers. Macromol. Rapid Commun. 2000, 21, 16-34.

(13) Asai, Y.; Takano, A.; Matsushita, Y. Creation of Cylindrical Morphologies with Extremely Large Oblong Unit Lattices from ABC Block Terpolymer Blends. Macromolecules 2015, 48, 1538-1542.

(14) Asai, Y.; Suzuki, J.; Aoyama, Y.; Nishioka, H.; Takano, A.; Matsushita, Y. Tricontinuous Double Diamond Network Structure from Binary Blends of $\mathrm{ABC}$ Triblock Terpolymers. Macromolecules 2017, 50, 5402-5411.

(15) Haenelt, T. G.; Abetz, C.; Abetz, V. Four-Phase Morphologies in Blends of ABC and BAC Triblock Terpolymers. Macromol. Chem. Phys. 2018, 219, 1700241.

(16) Hofman, A. H.; ten Brinke, G.; Loos, K. Hierarchical structure formation in supramolecular comb-shaped block copolymers. Polymer 2016, 107, 343-356.

(17) Ruokolainen, J.; ten Brinke, G.; Ikkala, O.; Torkkeli, M.; Serimaa, R. Mesomorphic Structures in Flexible Polymer-Surfactant Systems Due to Hydrogen Bonding: Poly(4-vinylpyridine)-Pentadecylphenol. Macromolecules 1996, 29, 3409-3415.

(18) Hofman, A. H.; Chen, Y.; ten Brinke, G.; Loos, K. Interaction Strength in Poly(4-vinylpyridine)-n-Alkylphenol Supramolecular Comb-Shaped Copolymers. Macromolecules 2015, 48, 1554-1562.

(19) Ruokolainen, J.; Mäkinen, R.; Torkkeli, M.; Mäkelä, T.; Serimaa, R.; ten Brinke, G.; Ikkala, O. Switching Supramolecular Polymeric Materials with Multiple Length Scales. Science 1998, 280, $557-560$.

(20) Ruokolainen, J.; ten Brinke, G.; Ikkala, O. Supramolecular Polymeric Materials with Hierarchical Structure-Within-Structure Morphologies. Adv. Mater. 1999, 11, 777-780. 
(21) Vukovic, I.; Voortman, T. P.; Hermida-Merino, D.; Portale, G.; Hiekkataipale, P.; Ruokolainen, J.; ten Brinke, G.; Loos, K. Double Gyroid Network Morphology in Supramolecular Diblock Copolymer Complexes. Macromolecules 2012, 45, 3503-3512.

(22) Gobius du Sart, G.; Vukovic, I.; Alberda van Ekenstein, G.; Polushkin, E.; Loos, K.; ten Brinke, G. Self-Assembly of Supramolecular Triblock Copolymer Complexes. Macromolecules 2010, 43, 2970-2980.

(23) Hiekkataipale, P.; Löbling, T. I.; Poutanen, M.; Priimagi, A.; Abetz, V.; Ikkala, O.; Gröschel, A. H. Controlling the shape of Janus nanostructures through supramolecular modification of $\mathrm{ABC}$ terpolymer bulk morphologies. Polymer 2016, 107, 456-465.

(24) Faber, M.; Hofman, A. H.; Polushkin, E.; Alberda van Ekenstein, G.; Seitsonen, J.; Ruokolainen, J.; Loos, K.; ten Brinke, G. Hierarchical self-assembly in supramolecular double-comb diblock copolymer complexes. Macromolecules 2013, 46, 500-517.

(25) Hofman, A. H.; Reza, M.; Ruokolainen, J.; ten Brinke, G.; Loos, K. Hierarchical Self-Assembly of Symmetric Supramolecular DoubleComb Diblock Copolymers: a Comb Density Study. Macromolecules 2014, 47, 5913-5925.

(26) Hofman, A. H.; Reza, M.; Ruokolainen, J.; ten Brinke, G.; Loos, K. Hierarchical Layer Engineering Using Supramolecular DoubleComb Diblock Copolymers. Angew. Chem., Int. Ed. 2016, 55, 1308113085 .

(27) Hofman, A. H.; Reza, M.; Ruokolainen, J.; ten Brinke, G.; Loos, K. The Origin of Hierarchical Structure Formation in Highly Grafted Symmetric Supramolecular Double-Comb Diblock Copolymers. Macromol. Rapid Commun. 2017, 38, 1700288.

(28) Hofman, A. H.; ten Brinke, G.; Loos, K. Asymmetric supramolecular double-comb diblock copolymers: From plasticization, to confined crystallization, to breakout. Polymer 2017, 121, 312319.

(29) Hofman, A. H.; Alberda van Ekenstein, G. O. R.; Woortman, A. J. J.; ten Brinke, G.; Loos, K. Poly(4-vinylpyridine)-block-poly(Nacryloylpiperidine) diblock copolymers: synthesis, self-assembly and interaction. Polym. Chem. 2015, 6, 7015-7026.

(30) Mogi, Y.; Kotsuji, H.; Kaneko, Y.; Mori, K.; Matsushita, Y.; Noda, I. Preparation and Morphology of Triblock Copolymers of the ABC Type. Macromolecules 1992, 25, 5408-5411.

(31) Bailey, T. S.; Pham, H. D.; Bates, F. S. Morphological Behavior Bridging the Symmetric $\mathrm{AB}$ and $\mathrm{ABC}$ States in the Poly(styrene- $b$ isoprene- $b$-ethylene oxide) Triblock Copolymer System. Macromolecules 2001, 34, 6994-7008.

(32) Borsboom, M.; Bras, W.; Cerjak, I.; Detollenaere, D.; Glastra van Loon, D.; Goedtkindt, P.; Konijnenburg, M.; Lassing, P.; Levine, Y. K.; Munneke, B.; Oversluizen, M.; van Tol, R.; Vlieg, E. The Dutch-Belgian beamline at the ESRF. J. Synchrotron Radiat. 1998, 5, 518-520.

(33) Bras, W.; Dolbnya, I. P.; Detollenaere, D.; van Tol, R.; Malfois, M.; Greaves, G. N.; Ryan, A. J.; Heeley, E. Recent experiments on a combined small-angle/wide-angle X-ray scattering beam line at the ESRF. J. Appl. Crystallogr. 2003, 36, 791-794.

(34) Liu, M.; Li, W.; Qiu, F.; Shi, A. Theoretical Study of Phase Behavior of Frustrated ABC Linear Triblock Copolymers. Macromolecules 2012, 45, 9522-9530.

(35) Betthausen, E.; Dulle, M.; Hanske, C.; Müller, M.; Fery, A.; Förster, S.; Schacher, F. H.; Müller, A. H. E. Nanoporous Sheets and Cylinders via Bulk Templating of Triblock Terpolymer/Homopolymer Blends. Macromolecules 2014, 47, 6289-6301.

(36) Radlauer, M. R.; Sinturel, C.; Asai, Y.; Arora, A.; Bates, F. S.; Dorfman, K. D.; Hillmyer, M. A. Morphological Consequences of Frustration in ABC Triblock Polymers. Macromolecules 2017, 50, 446-458.

(37) Polushkin, E.; Alberda van Ekenstein, G. O. R.; Knaapila, M.; Ruokolainen, J.; Torkkeli, M.; Serimaa, R.; Bras, W.; Dolbnya, I.; Ikkala, O.; ten Brinke, G. Intermediate Segregation Type Chain Length Dependence of the Long Period of Lamellar Microdomain Structures of Supramolecular Comb-Coil Diblocks. Macromolecules 2001, 34, 4917-4922.
(38) Alberda van Ekenstein, G. O. R.; Meyboom, R.; ten Brinke, G.; Ikkala, O. Determination of the Flory-Huggins Interaction Parameter of Styrene and 4-Vinylpyridine Using Copolymer Blends of Poly(styrene-co-4-vinylpyridine) and polystyrene. Macromolecules 2000, 33, 3752-3756.

(39) Asai, Y.; Takano, A.; Matsushita, Y. Asymmetric Double Tetragonal Domain Packing from ABC Triblock Terpolymer Blends with Chain Length Difference. Macromolecules 2016, 49, 6940-6946.

(40) Hardy, C. G.; Tang, C. Advances in Square Arrays Through Self-Assembly and Directed Self-Assembly of Block Copolymers. J. Polym. Sci., Part B: Polym. Phys. 2013, 51, 2-15.

(41) Tang, C.; Lennon, E. M.; Fredrickson, G. H.; Kramer, E. J.; Hawker, C. J. Evolution of Block Copolymer Lithography to Highly Ordered Square Arrays. Science 2008, 322, 429-432.

(42) Asai, Y.; Yamada, K.; Yamada, M.; Takano, A.; Matsushita, Y. Formation of Tetragonally-Packed Rectangular Cylinders from ABC Block Terpolymer Blends. ACS Macro Lett. 2014, 3, 166-169.

(43) Guliyeva, A.; Vayer, M.; Warmont, F.; Faugère, A. M.; Andreazza, P.; Takano, A.; Matsushita, Y.; Sinturel, C. Thin Films with Perpendicular Tetragonally Packed Rectangular Rods Obtained from Blends of Linear ABC Block Terpolymers. ACS Macro Lett. 2018, 7, 789-794.

(44) Chen, H.; Lu, J.; Yu, C.; Yeh, C.; Jeng, U.; Chen, W. Tetragonally Packed Cylinder Structure via Hierarchical Assembly of Comb-Coil Diblock Copolymer. Macromolecules 2007, 40, 32713276.

(45) Chiang, W.; Lin, C.; Yeh, C.; Nandan, B.; Hsu, P.; Lin, C.; Chen, H.; Chen, W. Tetragonally Packed Cylinder Structure of Comb-Coil Block Copolymer Bearing Heteroarm Star Architecture. Macromolecules 2009, 42, 2304-2308.

(46) Chuang, W.; Sheu, H.; Jeng, U.; Wu, H.; Hong, P.; Lee, J. Tetragonally Perforated Layer Structure via Columnar Ordering of 4'(3,4,5-Trioctyloxybenzoyloxy)benzoic Acid in a Supramolecular Complex with Polystyrene-block-Poly(4-vinylpyridine). Chem. Mater. 2009, 21, 975-978.

(47) Ludwigs, S.; Böker, A.; Abetz, V.; Müller, A. H. E.; Krausch, G. Phase behavior of linear polystyrene-block-poly(2-vinylpyridine)block-poly(tert-butyl methacrylate) triblock terpolymers. Polymer 2003, 44, 6815-6823.

(48) Valkama, S.; Ruotsalainen, T.; Nykänen, A.; Laiho, A.; Kosonen, H.; ten Brinke, G.; Ikkala, O.; Ruokolainen, J. SelfAssembled Structures in Diblock Copolymers with Hydrogen-Bonded Amphiphilic Plasticizing Compounds. Macromolecules 2006, 39, 9327-9336.

(49) Tillmann, S. D.; Hermida-Merino, D.; Winter, M.; CekicLaskovic, I.; Loos, K. Nanoporous polymer foams derived from high molecular PS- $b$-P4VP(PDP $)_{x}$ for template-directed synthesis approaches. RSC Adv. 2016, 6, 52998-53003. 